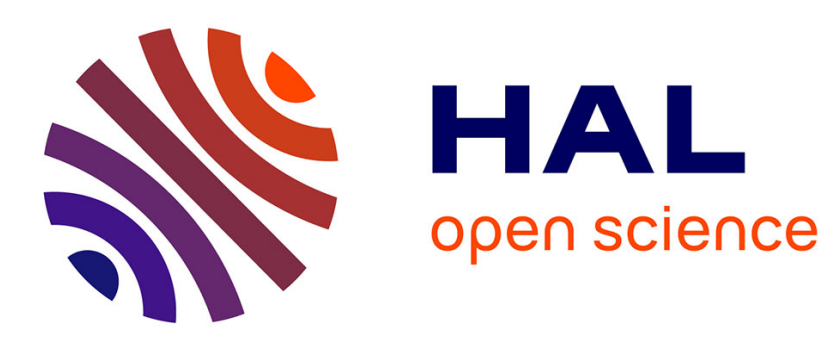

\title{
Geostatistical Simulations on Irregular Reservoir Models Using Methods of Nonlinear Geostatistics
}

\author{
Victor Zaytsev, Pierre Biver, Hans Wackernagel, Denis Allard
}

\section{To cite this version:}

Victor Zaytsev, Pierre Biver, Hans Wackernagel, Denis Allard. Geostatistical Simulations on Irregular Reservoir Models Using Methods of Nonlinear Geostatistics. Petroleum Geostatistics (EAGE), European Association of Petroleum Geologists, Sep 2015, Biarritz, France. hal-01258794

HAL Id: hal-01258794

https://hal-mines-paristech.archives-ouvertes.fr/hal-01258794

Submitted on 19 Jan 2016

HAL is a multi-disciplinary open access archive for the deposit and dissemination of scientific research documents, whether they are published or not. The documents may come from teaching and research institutions in France or abroad, or from public or private research centers.
L'archive ouverte pluridisciplinaire HAL, est destinée au dépôt et à la diffusion de documents scientifiques de niveau recherche, publiés ou non, émanant des établissements d'enseignement et de recherche français ou étrangers, des laboratoires publics ou privés. 


\title{
We B01
}

\section{Geostatistical Simulations on Irregular Reservoir Models Using Methods of Nonlinear Geostatistics}

\author{
V.N. Zaytsev* (Total / MINES ParisTech), P. Biver (Total), H. Wackernagel \\ (MINES ParisTech) \& D. Allard (INRA)
}

\section{SUMMARY}

Classical approaches to geostatistical simulations are not applicable directly on irregular reservoir models (such as Voronoi polygon and tetrahedron meshed models). One of the main difficulties is that the block marginal distributions are unique for every block due to volume support effect. We propose a methodology for geostatistical simulations which overcomes this difficulty in an analytical manner and provides a robust utilization of the small support petrophysical property distribution and the covariance model for irregular reservoir models. The proposed solution is based on the discrete Gaussian (DGM) model and operates directly on blocks of the target grid. This solution is also capable to improve the quality of the classical reservoir models, such as tartan meshes, by including the volume support effect into consideration and thus-providing geologically more realistic results. Applications to Voronoi polygon grid with local grid refinements and to a tartan-meshed offshore gas reservoir model are demonstrated. 


\section{Introduction}

Geostatistical simulation on irregular grids is a challenging task, since the algorithms face a multiscale problem posed by the transition from the small scale core analysis and seismic data to the multiple uneven supports of the grid cells. Classical sequential Gaussian simulation (SGS) is not applicable on irregular support, since the data does not average linearly after the normal score transform.

One approach to overcome this difficulty is using direct sequential simulation (DSS) algorithms, which avoid the transformation to the space of Gaussian variables and work directly in the space of the simulated variable (Manchuk et al. 2005, Soares 2001). Another approach for simulating on irregular support with the methods of non-linear geostatistics was proposed by Brown et al. (2008). The authors demonstrate the application of discrete Gaussian model (DGM) for simulating the Cox process using multiple sample supports representing the samples as a tree graphical model and using an extended conditional independence assumption. The solution is based on a theoretical model proposed by Emery (2007) for conditional simulations on regular support using DGM.

In this paper we provide an extension of the DGM-based geostatistical simulation algorithm for petroleum reservoirs, which uses the small support covariance derived from the data analysis and approximates with high accuracy the block-support marginal distributions. We first present two methods of transforming the problem of simulation on an irregular grid to the problem of simulating multivariate Gaussian random vectors. Then we demonstrate an application for simulating porosity on a tartan-meshed offshore gas field with significant volumetric differences between the cells.

\section{Discrete Gaussian model for geostatistical simulations}

Consider the problem of simulating on an irregular grid $\left\{v_{p}, p=1 \ldots N\right\}$ the volumetric averages $Z\left(v_{p}\right)=\frac{1}{\left|v_{p}\right|} \int_{v_{p}} Z(x) d x$ of the point-support random variable (RV) $Z(x)$ with a covariance function $C\left(x, x^{\prime}\right)$. Without additional assumptions, the marginal distribution of $Z\left(v_{p}\right)$ cannot be determined analytically from the distribution of $Z(x)$ and a Monte Carlo approach is applied, which in this context is referred to as mini-model simulation.

In case of irregular grids, the mini-model approach is hardly applicable, since it requires multiple fine scale simulations for every block of the model. The proposed simulation model enables to derive the blocks marginal distributions in an analytical form by introducing additional theoretical assumptions. The model assumes that $Z(x)$ is a transformation of a standard Gaussian RV $Y(x)$ with a covariance function $\rho\left(x, x^{\prime}\right)$ and that every block value $Z\left(v_{p}\right)$ is a block-dependent transformation of a standard Gaussian $\operatorname{RV} Y_{v_{p}}$

$$
Z(x)=\varphi(Y(x)) \text { and } Z\left(v_{p}\right)=\varphi_{v_{p}}\left(Y_{v_{p}}\right), p=1 \ldots N
$$

The transform function $\varphi(y)$ can be fitted to the core sample values and is considered to be known. The DGM approach enables to shift from the problem of simulating the set of $\operatorname{RV}\left\{Z\left(v_{p}\right), p=1 \ldots N\right\}$ to a problem of simulating a stationary multivariate Gaussian random vector $\left(Y_{v_{1}}, \ldots, Y_{v_{N}}\right)$ and applying appropriate transformation functions $\varphi_{v_{p}}, p=1 \ldots N$. The DGM in the strong form (DGM 1, see Chilès (2014) for discussion) can be established with the following two assumptions:

i. The vector $\left(Y_{v_{1}}, \ldots, Y_{v_{N}}\right)$ is multivariate Gaussian;

ii. For every block $v_{\mathrm{p}}$ the joint distribution of $Y_{v_{p}}$ and $Y(\underline{x})$ within $v_{p}$ is bivariate Gaussian with correlation coefficient $r_{p}$ (where $\underline{\mathrm{x}}$ denotes a uniformly random point within a block).

The block-dependent transform function $\varphi_{v_{p}}(y)$ can be determined from the change of support coefficient $r_{p}$ for block $v_{p}$ and the decomposition of $\varphi(y)$ in the basis of normalized Hermite polynomials $\left\{\chi_{i}(y), i=0 \ldots+\infty\right\}$ (Chilès and Delfiner 2012)

$$
\varphi(y)=\sum_{i=0}^{\infty} \varphi_{i} \chi_{i}(y) \Rightarrow \varphi_{v_{p}}(y)=\sum_{i=0}^{\infty} \varphi_{i} r_{v_{p}}^{i} \chi_{i}(y)
$$

Where the change of support coefficient $r_{p}$ for every block is defined by the equation 


$$
\operatorname{Var}\left(Z_{v_{p}}\right)=\frac{1}{|v|^{2}} \int_{v} \int_{v} C\left(x, x^{\prime}\right) d x d x^{\prime}=\sum_{i=1}^{\infty} \varphi_{i}^{2} r_{p}^{2 i}
$$

And the covariance matrix for $\left(Y_{v_{1}}, \ldots Y_{v_{N}}\right)$ is defined by equations

$$
\frac{1}{\left|v_{p}\right|\left|v_{q}\right|} \int_{v_{p}} \int_{v_{q}} C\left(x, x^{\prime}\right) d x d x^{\prime}=\sum_{i=1}^{\infty} \varphi_{i}^{2} r_{p}{ }^{i} r_{q}^{i} \operatorname{cov}\left(Y_{v_{p}}, Y_{v_{q}}\right)^{i}
$$

The multivariate Gaussian random vector $\left(Y_{v_{1}}, \ldots Y_{v_{N}}\right)$ can then be simulated using standard techniques, for instance SGS (Chilès and Delfiner 2012). Applying the transformation functions $\varphi_{v_{p}}(y), p=1 \ldots N$ gives an unconditional simulation of block average values $\left\{Z\left(v_{p}\right), p=1 \ldots N\right\}$ honoring the covariance function $C\left(x, x^{\prime}\right)$ and approximating with high accuracy the block marginal distributions (Chilès 2014, Chilès and Delfiner 2012, Matheron 1985). The simulated unconditional field can then be conditioned using block or point-support samples through simple or ordinary kriging and uniform conditioning (de Fouquet 1994).

Another convenient way of applying DGM for geostatistical simulations consists in introducing an additional assumption, which leads to a weak DGM model - DGM 2

iii. for any two blocks $v_{p}$ and $v_{q}$, the joint distribution of $Y(\underline{x}), \underline{x} \in v_{p}$ and $Y\left(\underline{x^{\prime}}\right), \underline{x}^{\prime} \in v_{q}$ is bivariate Gaussian.

Introducing this additional assumption enables a simpler way of deriving the block-support coefficients $r_{p}$ and the correlation between the components of $\left(Y_{v_{1}}, \ldots Y_{v_{N}}\right)$ through the covariance $\rho\left(x, x^{\prime}\right)$ of $Y(x)$ as demonstrated by Emery (2007)

$$
\begin{gathered}
r_{p}^{2}=\frac{1}{\left|v_{p}\right|\left|v_{p}\right|} \int_{v_{p}} \int_{v_{p}} \rho\left(x, x^{\prime}\right) d x d x^{\prime} \\
\operatorname{cov}\left(Y_{v_{p}}, Y_{v_{q}}\right)=\frac{1}{r_{p} r_{q}} \frac{1}{\left|v_{p}\right|\left|v_{q}\right|} \int_{v_{p}} \int_{v_{q}} \rho\left(x, x^{\prime}\right) d x d x^{\prime}
\end{gathered}
$$

DGM 2 enables establishing the simulation model in a simpler manner relative to DGM 1 by the cost of introduction a small bias into the model. Formally, using DGM 2 for simulation does not guarantee the correct reproduction of the covariance of the random vector $\left(Z\left(v_{1}\right), \ldots Z\left(v_{N}\right)\right)$ due to assumption iii which is discussed by Chilès (2014).

\section{Difference between DGM 1 and DGM 2}

In order to demonstrate the difference between DGM 1 and DGM 2, we provide statistical analysis of 100000 unconditional simulations on a Voronoi polygon 2D grid with 10 local grid refinements. The grid size is $20 \times 20 \mathrm{~km}^{2}$ with a vast diversity of block sizes demonstrated on Figure 1.
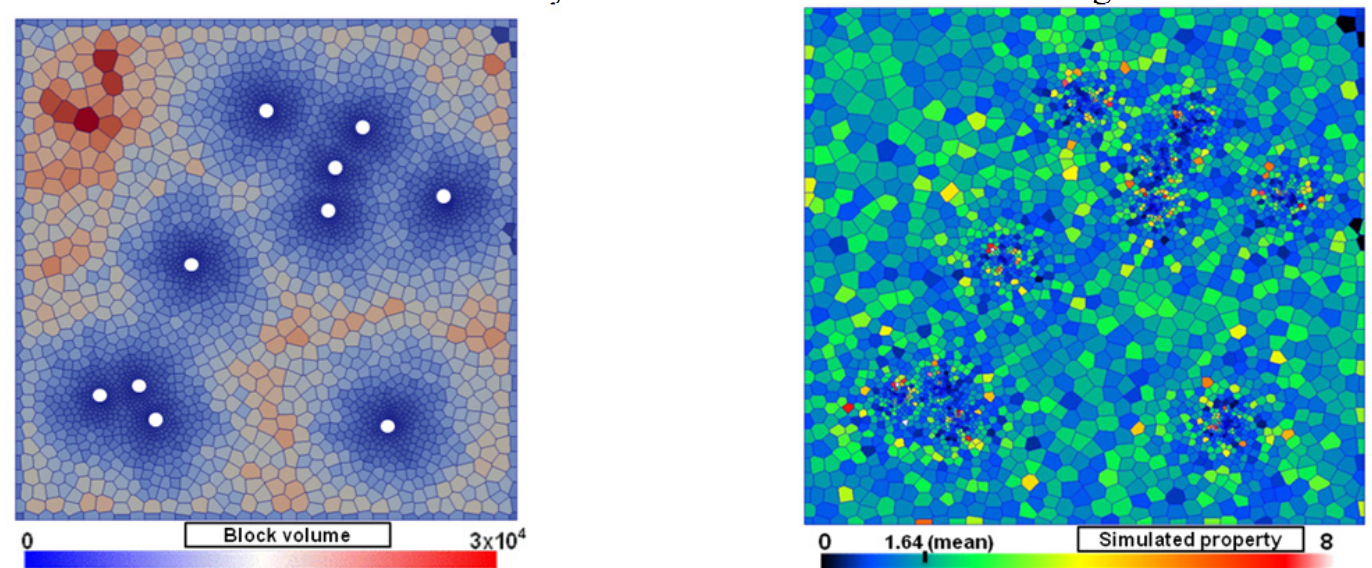

Figure 1 Voronoi-meshed 2D grid with 10 local grid refinements. Left) The block volumes and the well locations on the studied grid. Right) A simulation produced by DGM 2.

The grid contains 3546 blocks; the smallest and the largest block sizes are $36 \times 42$ and $1035 \times 1052 \mathrm{~m}$ respectively. The simulated variable $Z(x)$ has a standard lognormal distribution with logarithmic 


\section{PETROLEUM 2015 \\ GEOSTATISTICS}

parameters $(0,1)$ and the covariance $\rho\left(x, x^{\prime}\right)$ of the normal score transform variable $Y(x)$ is isotropic spherical with range $250 \mathrm{~m}$.

In order to check the accuracy of DGM 1 and DGM 2, we compare the block marginal distributions implied by these models with the results of 50000 Monte Carlo simulations on $2 \times 2 \mathrm{~m}^{2}$ support. The difference between the observed block variance and the theoretical variance is also illustrated (Figure $2)$. It reveals that DGM 2 introduces a non-linear bias into the block distribution variance, which, however, is below $5 \%$ of the point support variance of $Z(x)$.
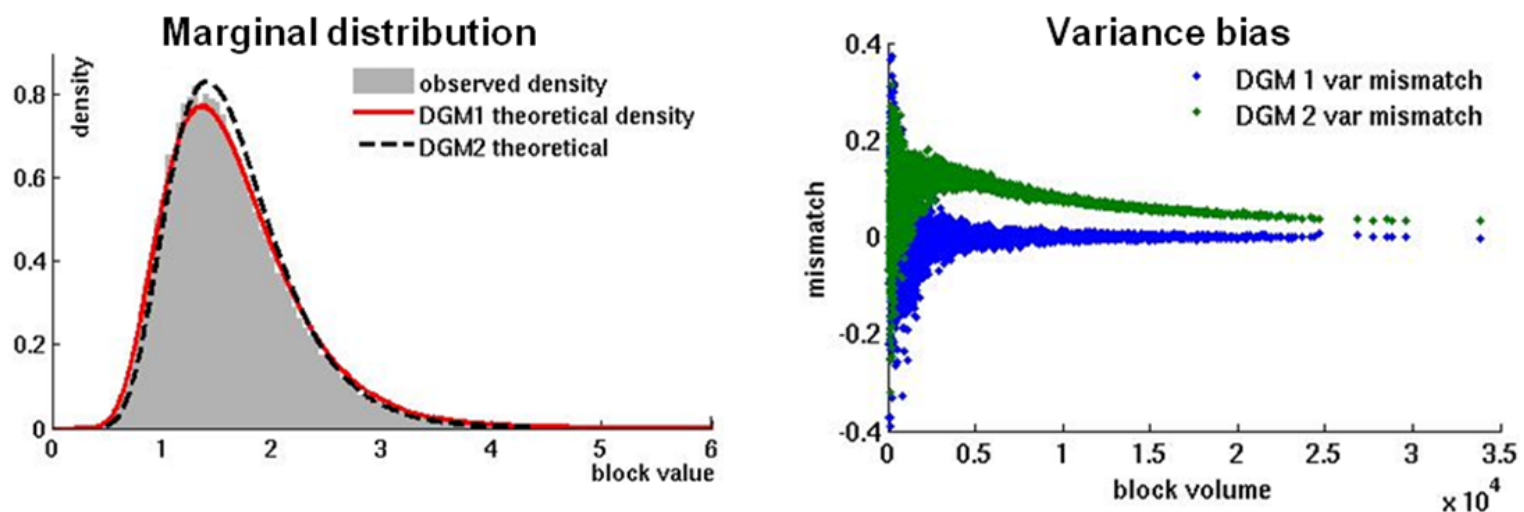

Figure 2 Quality check for DGM 1 and DGM 2. Left) The distribution for the largest block in the model. Right) The observed mismatch of the block variance versus block volume.

\section{Simulation of porosity on an offshore field tartan grid}

Addressing the change of support problem in geostatistical algorithms not only provides a solution for populating fully unstructured grids (such as Voronoi and tetrahedron meshing) but also enables to ameliorate the simulation results for classical tartan grids. Consider a tartan mesh of an offshore gas field $\mathrm{X}$ with dimensions $140 \times 200 \times 2 \mathrm{~km}^{3}$ (Figure 3).
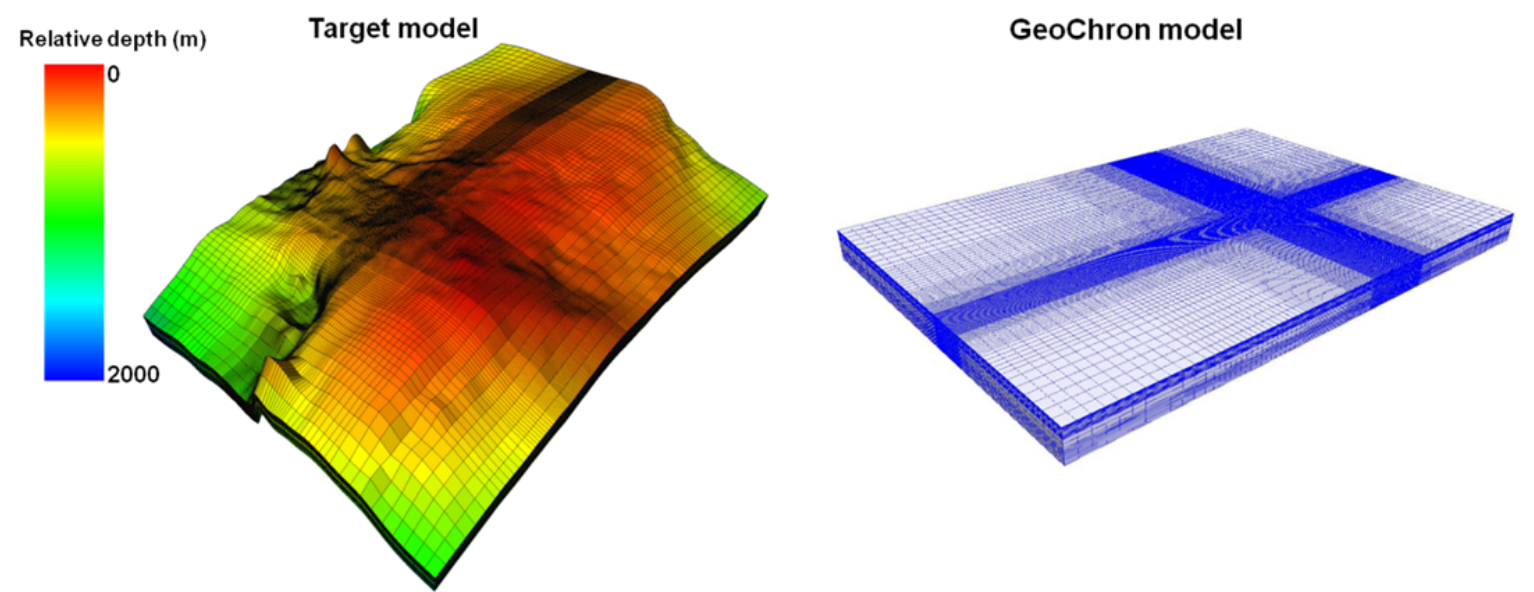

Figure 3 Tartan meshed offshore gas field model. Left) Original model. Right) Corresponding GeoChron model.

The classical approach consists of defining an average support for the model and the marginal distribution of porosity on this average support using Monte-Carlo simulations. The classical simulation proceeds in the $\boldsymbol{I J K}$ space, which leads to incorrect reproduction of block distributions and covariance. In order to treat the volume support effect in the simulation process, a proper simulation space is required in which the hypothesis of the stationary covariance model is applicable and the meshing structure of the reservoir is preserved. We adopt the GeoChron model (Mallet 2014) corresponding to the target reservoir meshed model to perform DGM-based simulations (Figure 3). 

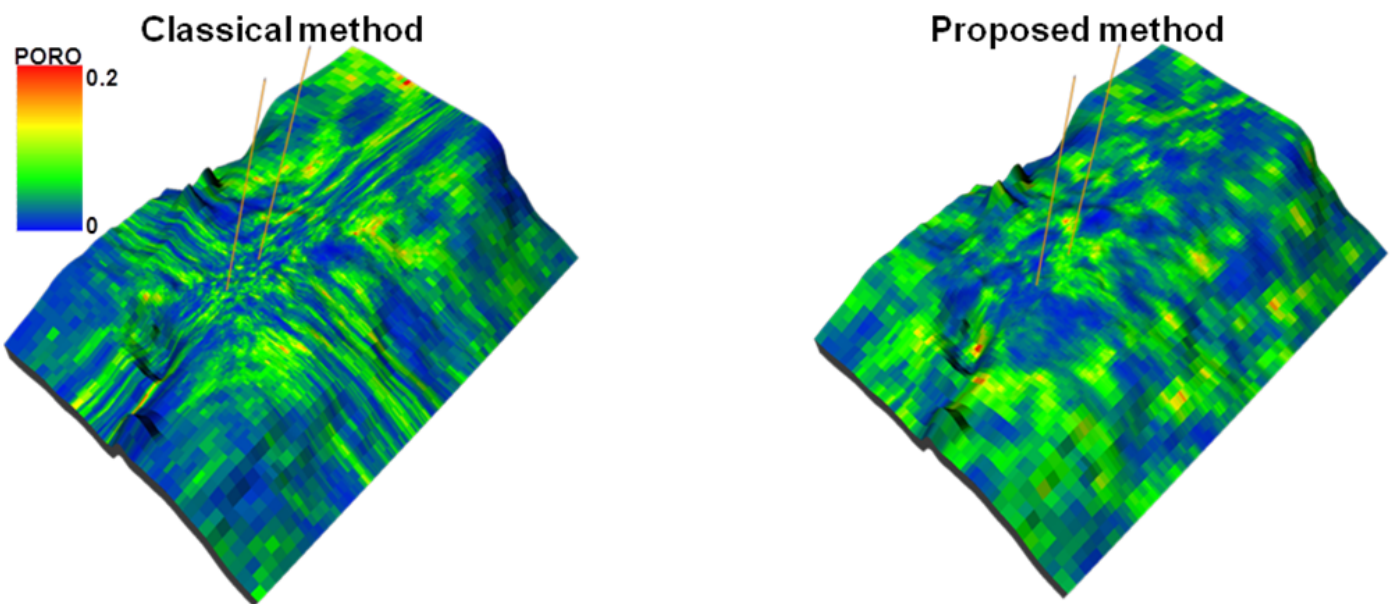

Figure 4 Result comparison. Left) Classical simulation in IJK on "average support". Right) Simulation in GeoChron model with DGM-based algorithm.

\section{Conclusions}

We propose a framework for geostatistical simulations on irregular reservoir grids using DGM. The proposed framework enables exact integration of the point-support covariance model on irregular support and approximating with high accuracy the block marginal distributions. It also enables to avoid the usage of the "average support" concept for simulations and to work on the target mesh directly. In addition, the proposed approach eliminates the artefacts imposed by the mesh, providing geologically more realistic full-size models.

\section{References}

Brown, G., Ferreira, J. and Lantuéjoul, C. [2012] Conditional simulation of a Cox process using multiple sample supports. $8^{\text {th }}$ International Geostatistics Congress, 459-468.

Chilès, J.-P. [2014] Validity range of the discrete Gaussian change-of-support model and its variant. Journal of the Southern African Institute of Mining and Metallurgy, 114(3), 231-235.

Chilès, J.-P. and Delfiner, P. [2012] Geostatistics: Modeling Spatial Uncertainty. Wiley.

de Fouquet, C. [1994] Reminders on the conditioning kriging. Geostatistical Simulations, 131-145. Springer Netherlands.

Emery, X. [2007] On some consistency conditions for geostatistical change-of-support models. Mathematical geology, 39(2), 205-223.

Mallet, J.-L. [2014] Elements of Mathematical Sedimentary Geology: the GeoChron Model. EAGE Publications bv.

Manchuk, J., Leuangthong, O. and Deutsch, C.V. [2005] Direct Geostatistical Simulation on Unstructured Grids. Geostatistics Banff 2004, 85-94. Springer Netherlands.

Matheron, G. [1985] Change of Support for Diffusion-Type Random Functions. Journal of the International Association for Mathematical Geology, 17(2), 137-165.

Soares, A. [2001] Direct sequential simulation and cosimulation. Mathematical Geology, 33(8), 911926. 